\section{Simultaneous Stabilization via Static Output Feedback and State Feedback}

Yong-Yan Cao, You-Xian Sun, and James Lam

\begin{abstract}
In this paper, the simultaneous stabilization problem is considered using the matrix inequality approach. Some necessary and sufficient conditions for simultaneous stabilizability of $\boldsymbol{r}$ strictly proper multi-input/multi-output (MIMO) plants via static output feedback and state feedback are obtained in the form of coupled ARI's. It is shown that any such stabilizing feedback gain is the solution of some coupled linear quadratic control problems where every cost functional has a suitable cross term. A heuristic iterative algorithm based on the linear matrix inequality (LMI) technique is presented to solve the coupled matrix inequalities. The effectiveness of the approach is illustrated by numerical examples.
\end{abstract}

Index Terms-Algebraic Riccati inequality, linear matrix inequality, simultaneous stabilization, static output feedback.

\section{INTRODUCTION}

Simultaneous stabilization is an important problem in the area of robust control design. It is the problem of determining a single controller which will simultaneously stabilize a finite collection of plants. It may apply to linear plants characterized by different modes of operation (for instance, failure modes) or to the stabilization of nonlinear plants linearized at several equilibria. Recently, research results also clearly show the relevance of simultaneous stabilization to system robustness [1].

The simultaneous stabilization problem was first introduced in [2] and [3]. It was shown that in the case of two plants, the problem reduces to the well-known strong stabilization problem. The problem of simultaneously stabilizing $r$ plants can always be reduced to the problem of simultaneously stabilizing $r-1$ plants using a stable compensator, as shown in [4]. Other design results on simultaneously stabilizing controllers can be found in [5]-[7]. However, even for three single-input/single-output (SISO) systems, no tractable simultaneous stabilization design procedure has been proposed. Basically, there are still many open problems associated with simultaneous stabilization.

Recently, Blondel and Gevers [8] discussed the complexity of simultaneous stabilization and proved that the simultaneous stabilizability of three linear systems is rationally undecidable. From their paper, one may conclude that this problem is very difficult due to its NP-hard nature (see also [26] and [27]). Fortunately, this does not signify the end of the simultaneous stabilization problem. One possible approach to deal with this kind of problem is to find an efficient numerical algorithm. It now becomes clear that an effective

Manuscript received January 27, 1997; revised December 3, 1997. Recommended by Associate Editor, H. Ozbay. The work of Y.-Y. Cao was supported in part by the Alexander von Humboldt Foundation. This work was supported in part by the National Natural Science Foundation of China under Grant 69604007 and an HKU CRCG Grant.

Y.-Y. Cao was with the Department of Measurement and Control, Duisburg University, 47048 Duisburg, Germany. He is now with the Department of Measurement and Control, Duisburg University, Germany (e-mail: h1218yo@unidui.uni-duisburg.de).

Y.-X. Sun is with the Institute of Industrial Process Control of Zhejiang University, Hangzhou, 310027 China.

J. Lam is with the Department of Mechanical Engineering, University of Hong Kong.

Publisher Item Identifier S 0018-9286(99)03029-9. approach to tackle a variety of simultaneous stabilization problems is through numerical means. In general, nonsmooth optimization techniques have to be used. Some research effort has been directed along this line of thought, such as [9]-[13]. Unfortunately, an efficient algorithm for this problem is as yet to be constructed. An alternative approach is to reduce this kind of problem to a well-known computation problem. In this paper, we will focus on reducing the simultaneous stabilization problem to a computational procedure of iterative nature.

The purpose of this paper is to construct a numerical algorithm to determine the existence of a simultaneously stabilizing static output feedback controller for a collection of given linear time-invariant multi-input/multi-output (MIMO) plants using matrix inequality approach. We hasten to note that the matrix inequality approach has already been employed for the simultaneous stabilization problem via state feedback by Geromel et al. [13]. However, the simultaneous stabilization via static output feedback has not been dealt with in that context. The main contribution of this paper can be summarized as follows. It is shown that there exists a simultaneously stabilizing controller via static output feedback or state feedback if and only if there exists a stabilizing solution for a collection of coupled algebraic Riccati inequalities (ARI's) or a collection of coupled linear quadratic (LQ) control problems in which the cost functional has a suitable cross term satisfying an inequality constraint. The solution procedure is applicable to any fixed number of plants. The design strategy consists of finding suitable weighting matrices such that the solution of these coupled LQ control problems corresponds to a simultaneously stabilizing output feedback controller. A heuristic iterative algorithm based on the linear matrix inequality (LMI) technique is presented to solve the weighting matrices and generate the feedback gain. It should be pointed out that the idea of this paper derives from [19]. However, the numerical algorithm presented here is novel and may also be employed to solve the problem in [19] and [24].

\section{PRELIMINARIES}

In this paper, all matrices are with appropriate dimensions if the dimensions are not explicitly stated. The notation $X>0$ means that $X$ is a symmetric and positive definite matrix.

First, let us consider the linear time-invariant plant $G$ described by the equation

$$
x(t)=A x(t)+B u(t), \quad x(0)=x_{0}
$$

where $x(t) \in \mathrm{R}^{n}$ is the state vector, $u(t) \in \mathrm{R}^{m}$ is the control vector, and $A, B$ are constant matrices. The following lemma is well-known [14], [15].

Lemma 1: Let the plant $G$ be given by (1). Then $G$ is stabilizable via state feedback if and only if there exist matrices $P>0, Q>0$, and $R>0$ satisfying the following algebraic Riccati equation (ARE):

$$
P A+A^{T} P-P B R^{-1} B^{T} P+Q=0
$$

or equivalently, there exist matrices $P>0$ and $R>0$ satisfying the following ARI:

$$
P A+A^{T} P-P B R^{-1} B^{T} P<0 .
$$

Remark 1: In fact, $(A, B)$ is stabilizable, if and only if, for any $Q>0$ and $R>0$ the above ARE has a unique solution $P>0$; see [15], or equivalently, for any $R>0$, the above ARI has a feasible solution; see [14], [20], and [21]. 
The LQ control problem with cross term, which we take as the basis for our development, involves minimizing the cost

$$
v\left(x_{0}\right)=\int_{0}^{\infty}\left(x^{T} Q x+2 u^{T} S x+u^{T} R u\right) d t
$$

where $Q>0, R>0$, and $S$ are constant weighting matrices satisfying

$$
Q-S^{T} R^{-1} S>0 .
$$

The solution of the LQ control problem associated with (1)-(3) is [15]

$$
\begin{gathered}
u=K x, \quad K=-R^{-1}\left(B^{T} P+S\right) \\
P A+A^{T} P-\left(B^{T} P+S\right)^{T} R^{-1}\left(B^{T} P+S\right)+Q=0 .
\end{gathered}
$$

Lemma 2: Let the plant $G$ be given by (1). Then $G$ is stabilizable via state feedback if and only if there exist matrices $P>0, Q>0$, $R>0$, and $S$ satisfying (3) and ARE (4) with cross term, or equivalently, there exist matrices $P>0, R>0$, and $S$ satisfying the following modified ARI with cross term:

$$
P A+A^{T} P-\left(B^{T} P+S\right)^{T} R^{-1}\left(B^{T} P+S\right)+S^{T} R^{-1} S<0 .
$$

Remark 2: Lemma 2 was established in [24]. Based on its proof in [24] and Lemma 1, it can be shown that for any $R>0$, ARI (5) has a feasible solution $(P, S)$ if and only if $(A, B)$ is stabilizable.

\section{A NECESSARY AND SufFICIENT CONDITION}

Now we consider the simultaneous stabilization of $r$ plants $G_{i}$

$$
\dot{x}_{i}(t)=A_{i} x_{i}(t)+B_{i} u_{i}(t), \quad y_{i}(t)=C_{i} x_{i}(t)
$$

where the state $x_{i}(t) \in \mathrm{R}^{n}$, the input $u_{i}(t) \in \mathrm{R}^{m}$, the output $y_{i}(t) \in \mathrm{R}^{p}$ and $n$ is the order of $G_{i}$. We assume

$$
C_{i}=C, \quad i=1, \cdots, r
$$

and $C$ has full rank. For example, this condition is satisfied for singleoutput plants of the same order since it is always possible to realize them in minimal observable form. In addition, for multiple model control of $r$ models $G_{i}(s)$ of an uncertain plant, uncertainty often exists only in the system matrix and (or) input matrix. In these situations, (7) is satisfied. Let $E$ be the right inverse of $C$, i.e., $C E=I_{p}$ where $I_{p}$ is the identity matrix of order $p$. Since $C$ has full rank, $E$ can be constructed from

$$
E=C^{T}\left(C C^{T}\right)^{-1} \text {. }
$$

So $E_{\perp}=E C$ is the orthogonal projection matrix on $\operatorname{Im}\left(C^{T}\right)$ and $x_{\perp}=E_{\perp} x=E y$.

Theorem 1: Let $r$ plants $G_{i}$ be given by (6) with same output matrix $C$, then they are simultaneously stabilizable via static output feedback if and only if there exist matrices $P^{i}>0, R>0, Q^{i}>0$, and $M$ satisfying the following coupled ARE's:

$$
\begin{aligned}
& P^{i} A_{i}+A_{i}^{T} P^{i}-\left(S^{i}+B^{T} P^{i}\right)^{T} R^{-1}\left(S^{i}+B_{i}^{T} P^{i}\right)+Q^{i} \\
& \quad=0, \quad i=1, \cdots, r \\
& Q^{i}-S^{i^{T}} R^{-1} S^{i}>0 \\
& S^{i}=M E_{\perp}-B_{i}^{T} P^{i}
\end{aligned}
$$

or equivalently, there exist matrices $P^{i}>0, R>0$, and $M$ satisfying the following coupled ARI's:

$$
\begin{aligned}
& P^{i} A_{i}+A_{i}^{T} P^{i}-\left(S^{i}+B_{i}^{T} P^{i}\right)^{T} R^{-1}\left(S^{i}+B_{i}^{T} P^{i}\right) \\
& \quad+S^{i^{T}} R^{-1} S^{i}<0, \quad i=1, \cdots, r .
\end{aligned}
$$

Moreover, the simultaneously stabilizing static output feedback controller can be constructed from

$$
K=R^{-1} M C^{T}\left(C C^{T}\right)^{-1} .
$$

The proof is similar to that of [19] and [25]. Some subsequent corrections and comments may be found in [17], [23], and [24]. The necessary modification in the definition of $\alpha^{*}$ is that

$$
\alpha^{*}=\max _{i=1, \cdots, r}\left(\alpha_{i}^{*}\right)
$$

where

$$
\begin{aligned}
& \alpha_{i}^{*}=\max _{x} \frac{x^{T}\left(P^{i} A_{i}+A_{i}^{T} P^{i}\right) x}{x^{T}\left(E_{\perp} M^{T} R^{-1} R^{-1} M E_{\perp}\right)}, \\
& x \notin \operatorname{Ker}\left(M E_{\perp}\right), x \neq 0, i=1, \cdots, r .
\end{aligned}
$$

Another modification is that (10) is also a necessary condition as a consequence of Lemma 1. In [19] this fact has been ignored, hence the main result is invalid (see [24]).

This theorem implies that simultaneous stabilization via static output feedback can be viewed as the solution of $r$-coupled LQ control problems with suitable weighting matrices $Q^{i}, R$, and $S^{i}$ in the functional (2) satisfying the coupled constraint (11).

Corollary 1: Given the plant $G$ in (1), it is stabilizable via static output feedback if and only if there exist matrices $Q>0, R>0$, and $M$ such that the following constrained ARE:

$$
\begin{array}{r}
P A+A^{T} P-\left(S+B^{T} P\right)^{T} R^{-1}\left(S+B^{T} P\right)+Q=0 \\
Q-S^{T} R^{-1} S>0 \\
S=M E_{\perp}-B^{T} P
\end{array}
$$

has a solution $P>0$, or equivalently, there exist matrices $P>0$, $R>0$, and $M$ satisfying the following constrained ARI with suitable cross term

$$
P A+A^{T} P-\left(S+B^{T} P\right)^{T} R^{-1}\left(S+B^{T} P\right)+S^{T} R^{-1} S<0 .
$$

Remark 3: Corollary 1 is the corrected version of [19, Th. 3.1] (see [24]). By comparing Theorem 1 and Corollary 1 with Lemma 2, it can be observed that the range space of the cross matrix $S$ is constrained. In the case of static output feedback, constraint (16) appears, and so $M \in \mathrm{R}^{m \times p}$ becomes the free variable instead of $S \in \mathrm{R}^{m \times n} . S$ is related to the output matrix $C$ such that $S \in \operatorname{Range}(C)$. For the static output feedback simultaneous stabilization of $r$ plants, $M$ is the coupling variable. Obviously, this condition is not related to the number of the plants to be simultaneous stabilized. In [7], however, the number of plants is restricted by the dimensions of the input and output.

Corollary 2: Let $r$ plants $G_{i}$ be given by (6), then they are simultaneously stabilizable via state feedback if and only if there exist matrices $P^{i}>0, R>0, Q^{i}>0$, and $M$ satisfying the following coupled ARE's:

$$
\begin{gathered}
P^{i} A_{i}+A_{i}^{T} P^{i}-\left(S^{i}+B_{i}^{T} P^{i}\right)^{T} R^{-1}\left(S^{i}+B_{i}^{T} P^{i}\right)+Q^{i}=0 \\
i=1, \cdots, r \\
Q^{i}-S^{i^{T}} R^{-1} S^{i}>0 \\
S^{i}=M-B_{i}^{T} P^{i}
\end{gathered}
$$

or equivalently, there exist matrices $P_{i}>0, R>0$, and $M$ satisfying the following coupled ARI's:

$$
\begin{gathered}
P^{i} A_{i}+A_{i}^{T} P^{i}-\left(S^{i}+B_{i}^{T} P^{i}\right)^{T} R^{-1}\left(S^{i}+B_{i}^{T} P^{i}\right) \\
+S^{i^{T}} R^{-1} S^{i}<0, \quad i=1, \cdots, r .
\end{gathered}
$$


Remark 4: Note that Corollary 2 corresponds to the case where $C=I$ in Theorem 1 . An obvious advantage of the above approach is that the quadratic stability is not required to be satisfied [14], [16]. This is because a different plant is associated with a different Lyapunov matrix $P_{i}$. In general, the results are usually more conservative if quadratic stability is used to treat simultaneous stabilization.

\section{An Iterative LMI Algorithm}

In this section, we present an iterative algorithm based on the theory in earlier sections to simultaneously stabilize $r$ plants in (6) via static output feedback and state feedback control.

From the results in Section III, the static output feedback stabilization problem is transformed into solving (17) under constraint (16). Moreover, the static output (respectively, state) feedback simultaneous stabilization problem become the feasibility of inequalities (12) [respectively, (20)] under constraint (11) [respectively, (19)]. Obviously, as the constraints (11), (16), and (19) are all linear, the key to solving these problems lies in the solution of (5).

In fact, all of the above matrix inequality problems belong to the class of bilinear matrix inequality (BMI) problems, which are generally very difficult for which to obtain solutions or to determine feasibility [26]. However, if we can derive an iterative form for its feasibility, we may construct an iterative algorithm based on the LMI technique [22]. Inequality (5) is equivalent to the following nonlinear matrix inequality:

$$
P A+A^{T} P-P B R^{-1} B^{T} P-S^{T} R^{-1} B^{T} P-P B R^{-1} S<0 .
$$

From Remark 1 and the proof of Theorem 1, we may let $R=I$ so that (21) becomes a BMI problem. In fact, this selection is without loss of generality for the following reason. From the proof of Theorem 1 (see also [19] and [23]), it can be seen that if the plants are stabilizable simultaneously via static output feedback, then a sufficiently large $\alpha$ can be chosen so that when $R=\alpha I$, there exist matrices $P^{i}$ and $M$ that satisfy (12) and (11). Consequently, $\bar{P}^{i}=\alpha^{-1} P^{i}, \bar{S}^{i}=\alpha^{-1} S^{i}, \bar{M}^{i}=\alpha^{-1} M^{i}, \bar{R}=I, i=1, \cdots, r$, is also a solution of ARI's (12) constrained by (11). In other words, a feasible solution exists for BMI (21) with $R=I$. In fact, we have established the following result.

Corollary 3: Let $r$ plants $G_{i}$ be given by (6), then they are simultaneously stabilizable via static output feedback if and only if there exist matrices $P^{i}>0, Q^{i}>0$, and $M$ satisfying the following coupled ARE's:

$$
\begin{aligned}
& P^{i} A_{i}+A_{i}^{T} P^{i}-\left(S^{i}+B_{i}^{T} P^{i}\right)^{T}\left(S^{i}+B_{i}^{T} P^{i}\right)+Q^{i}=0, \\
& \quad i=1, \cdots, r \\
& Q^{i}-S^{i^{T}} S^{i}>0, \quad S^{i}=M E_{\perp}-B_{i}^{T} P^{i}
\end{aligned}
$$

or equivalently, there exist matrices $P^{i}>0$ and $M$ satisfying the following coupled ARI's:

$$
\begin{gathered}
P^{i} A_{i}+A_{i}^{T} P^{i}-\left(S^{i}+B_{i}^{T} P^{i}\right)^{T}\left(S^{i}+B_{i}^{T} P^{i}\right)+S^{i^{T}} S^{i}<0, \\
\quad i=1, \cdots, r .
\end{gathered}
$$

Theorem 2: There exists a feasible solution $(P>0, S)$ satisfying the BMI (21) if and only if the following matrix inequality holds:

$P A+A^{T} P-2 P B R^{-1} B^{T} P+\left(B^{T} P-S\right)^{T} R^{-1}\left(B^{T} P-S\right)<0$.

$$
\begin{aligned}
& \text { Proof-Sufficiency: Note that } \\
& \begin{aligned}
P A+ & A^{T} P-P B R^{-1} B^{T} P-S^{T} R^{-1} B^{T} P-P B R^{-1} S \\
\leq & P A+A^{T} P-P B R^{-1} B^{T} P-S^{T} R^{-1} B^{T} P \\
& \quad-P B R^{-1} S+S^{T} R^{-1} S \\
= & P A+A^{T} P-2 P B R^{-1} B^{T} P+\left(B^{T} P-S\right)^{T} R^{-1} \\
& \cdot\left(B^{T} P-S\right)<0 .
\end{aligned}
\end{aligned}
$$

Necessity: It is not difficult to see that there must exist a scalar $\rho>1$ such that

$$
\begin{aligned}
P A & +A^{T} P-P B R^{-1} B^{T} P-S^{T} R^{-1} B^{T} P-P B R^{-1} S \\
& +\rho^{-2} S^{T} R^{-1} S<0
\end{aligned}
$$

i.e.,

$$
\begin{aligned}
P A+ & A^{T} P-\left(\rho^{2}+1\right) P B R^{-1} B^{T} P+\left(\rho B^{T} P-\rho^{-1} S\right)^{T} R^{-1} \\
& \cdot\left(\rho B^{T} P-\rho^{-1} S\right)<0 .
\end{aligned}
$$

Hence

$$
\begin{aligned}
P A+ & A^{T} P-2 \rho^{2} P B R^{-1} B^{T} P+\left(\rho B^{T} P-\rho^{-1} S\right)^{T} R^{-1} \\
& \cdot\left(\rho B^{T} P-\rho^{-1} S\right)<0 .
\end{aligned}
$$

Substituting $\rho^{2} P$ with $P$, we obtain (23).

Due to the negative sign in the $-2 P B R^{-1} B^{T} P$ term, (23) cannot be simplified to an LMI. To accommodate the $-2 P B R^{-1} B^{T} P$ term, we introduce an additional design variable $X$. Since $(X-$ $P)^{T} B R^{-1} B^{T}(X-P) \geq 0$ for any $X$ and $P$ of the same dimension, we obtain

$$
\begin{aligned}
& X^{T} B R^{-1} B^{T} P+P^{T} B R^{-1} B^{T} X-X^{T} B R^{-1} B^{T} X \\
& \quad \leq P^{T} B R^{-1} B^{T} P .
\end{aligned}
$$

The equality holds if $X=P$. This leads to a sufficient condition for the feasibility of the BMI (21)

$$
\begin{aligned}
& A^{T} P+P A-2 X B R^{-1} B^{T} P-2 P B R^{-1} B^{T} X \\
& \quad+2 X B R^{-1} B^{T} X+\left(B^{T} P-S\right)^{T} R^{-1}\left(B^{T} P-S\right)<0 .
\end{aligned}
$$

Theorem 3: There exists a feasible solution $(P>0, S)$ satisfying the BMI (21) if and only if there exist a matrix $S$ and two matrices $P>0$ and $X>0$ satisfying the matrix inequality (26).

Proof: The sufficiency is obvious, thus only the necessity needs to be proven. Obviously, if BMI (21) has a feasible solution $(P>$ $0, S)$, then there exists a positive real number $\varepsilon>0$ such that

$$
\begin{gathered}
A^{T} P+P A-2 P B R^{-1} B^{T} P+\left(B^{T} P-S\right)^{T} R^{-1} \\
\cdot\left(B^{T} P-S\right)+\varepsilon I<0 .
\end{gathered}
$$

Select a symmetric matrix $\bar{X} \geq 2 B R^{-1} B^{T}$ and set $X=P-\Delta X$, where $\Delta X=\varepsilon^{1 / 2} \bar{X}^{-1 / 2}$, then

$$
2(P-X) B R^{-1} B^{T}(P-X) \leq \varepsilon I .
$$

So (26) holds.

Using the Schur complement, (26) is equivalent to the quadratic matrix inequality shown in (27), at the bottom of the page. This BMI points to an iterative approach to solve the matrices $S$ and $P>0$, namely, if $X$ is fixed in (27), then it reduces to an LMI problem in the unknowns $S$ and $P$. The LMI problem is convex and can be solved if a feasible solution exists.

$$
\left[\begin{array}{cc}
A^{T} P+P A-2 X B R^{-1} B^{T}-2 P B R^{-1} B^{T} X+2 X B R^{-1} B^{T} X & \left(B^{T} P-S\right)^{T} \\
\left(B^{T} P-S\right) & -R
\end{array}\right]<0
$$


When $X$ is fixed, however, LMI (27) is only a sufficient condition for feasibility of BMI (21). In fact, if we find its solution, then we find a solution of (21). Unfortunately, it has no solution is general. On the other hand, if we simply perturb (26) by $-\beta P$, then we obtain a necessary condition for static output feedback stabilizability, i.e.,

$$
\begin{aligned}
& A^{T} P+P A-\beta P-2 X B R^{-1} B^{T} P-2 P B R^{-1} B^{T} X \\
& \quad+2 X B R^{-1} B^{T} X+\left(B^{T} P-S\right)^{T} R^{-1}\left(B^{T} P-S\right)<0 .
\end{aligned}
$$

Consequently, the closed-loop system matrices $A-B K C$ have eigenvalues on the left-hand side of the line $\Re(s)=\beta / 2$ in the complex $s$-plane. Based on the idea that all eigenvalues of $A-B K C$ are shifted progressively toward the left half-plane through the reduction of $\beta$, we may close in on the feasibility of (2). In the following algorithm, we will fix $R=I$.

Iterative Linear Matrix Inequality (ILMI) Algorithm:

Step 1) Set $i=1$, select $Q>0$. Solve the following ARE:

$$
A^{T} P+P A-P B B^{T} P+Q=0
$$

and set $X=P$.

Step 2) Solve the following optimization problem for $P_{i}, M$, and $\beta_{i}$.

OP1: Minimize $\beta_{i}$ subject to the LMI constraints shown in (28)-(30), shown at the bottom of the page.

Step 3) If $\beta_{i} \leq 0,\left(P_{i}, S\right)$ is a feasible solution. STOP.

Step 4) Solve the following optimization problem for $P_{i}$ and $M$.

OP2: Minimize trace $\left(P_{i}\right)$ subject to the LMI constraints shown in (28)-(30).

Step 5) If $\left\|X-P_{i}\right\|<\delta$, a predetermined tolerance, go to Step 6); else set $X=P_{i}$ and $i=i+1$, then go to Step 2).

Step 6) This algorithm cannot get a feasible solution. STOP.

Remark 5: The optimization problem in Step 2) is a generalized eigenvalue minimization problem. This step guarantees that the poles of the closed-loop system move to the left half-plane progressively. The optimization problem OP2 is necessary to guarantee the convergence of the algorithm. Numerical experiences indicated that $\beta$ may converge slowly in some cases. One way to terminate the algorithm is when $\beta_{i-1}-\beta_{i}$ is smaller than a prescribed tolerance for a fixed number of successive iterations. In Step 3), if a feasible solution is obtained and the feedback gain is too large, one may set $\beta_{i}=0$ and let the algorithm continue iterating to make the difference of $X$ and $P$ as small as possible.

Remark 6: Inequality (27) plays a crucial role in the ILMI algorithm. Obviously, there always exists a solution for the optimization problem OP1 when $i=1$. For $i>1$, the existence of the solution is guaranteed by (27). For a given stabilizable system (that is, (27) has a solution), the solution sequence $\beta_{i}$ is a decreasing sequence.
This is because if

$$
\begin{gathered}
A^{T} P_{i}+P_{i} A-2 X B B^{T} P_{i}-2 P_{i} B B^{T} X+2 X B B^{T} X \\
-\beta_{i} P_{i}+\left(B^{T} P_{i}-S\right)^{T}\left(B^{T} P_{i}-S\right)<0
\end{gathered}
$$

then

$A^{T} P_{i}+P_{i} A-2 P_{i} B B^{T} P_{i}-\beta_{i} P_{i}+\left(B^{T} P_{i}-S\right)^{T}\left(B^{T} P_{i}-S\right)<0$

a solution $\beta_{i+1} \leq \beta_{i}$ can be found in Step 2) with $P_{i+1}=$ $P_{i}, \beta_{i+1}=\beta_{i}$.

Remark 7: The existence of a solution of optimization problem OP2 is ensured by (28). The solution $P_{i}$ is a symmetric positivedefinite matrix which implies that the sequence $\operatorname{trace}\left(P_{i}\right)$ is bounded below. It is not difficult to find that the solution sequence $\operatorname{trace}\left(P_{i}\right)$ is a monotonic decreasing sequence if $\beta_{i}$ is fixed for $i>k$ and $k$ is a positive constant. Due to the effect of numerical errors in Step 2), optimization problem OP2 may be infeasible. In such a case, we set $\beta_{i}=\beta_{i}+\Delta \beta_{i}$ for some small positive number $\Delta \beta_{i}$ and solve OP2 again.

Remark 8: This algorithm can be easily extended to find a solution for the static output feedback simultaneous stabilization problem involving $r$ plants with the same output matrix $C$. In this case, (28)-(30) should be as shown in (31), at the bottom of the page, and in Step 4) $\operatorname{trace}\left(P_{i}\right)$ should be replaced by $\sum_{j=1}^{r} \operatorname{trace}\left(P_{i}^{j}\right)$. By duality, the static output feedback simultaneous stabilization of $r$ plants with same input matrix $B$ can also be considered.

\section{EXAMPLES}

Example 1: Consider the following linear parameter-varying plant:

$$
G(s)=\frac{s+\sqrt{\theta}+1}{s^{2}+\left(\theta^{2}-10\right) s+3 \theta+11}, \quad 3 \leq \theta \leq 11 .
$$

The nominal operating point is $\theta_{0}=7$. We consider simultaneously stabilizing three plants at operating points $\theta_{0}=7, \theta_{1}=3$, and $\theta_{2}=11$. The minimal realizations of these three plants are

$$
\begin{aligned}
& A_{0}=\left[\begin{array}{ll}
-39 & 1 \\
-32 & 0
\end{array}\right], \quad B_{0}=\left[\begin{array}{c}
1 \\
3.646
\end{array}\right], \quad A_{1}=\left[\begin{array}{cc}
1 & 1 \\
-20 & 0
\end{array}\right] \\
& B_{1}=\left[\begin{array}{c}
1 \\
2.732
\end{array}\right], \quad A_{2}=\left[\begin{array}{cc}
-111 & 1 \\
-44 & 0
\end{array}\right], \quad B_{2}=\left[\begin{array}{c}
1 \\
4.317
\end{array}\right] \\
& C_{0}=C_{1}=C_{2}=\left[\begin{array}{ll}
1 & 0
\end{array}\right] \text {. }
\end{aligned}
$$

Using the ILMI Algorithm, we obtain $\beta=-5.464$ and $K=$ $-1.9322 * 10^{7}$ only after one iteration. The feedback gain is unacceptably large and hence the algorithm is allowed to continue iterating.

$$
\begin{aligned}
& {\left[\begin{array}{cc}
A^{T} P_{i}+P_{i} A-2 X B B^{T} P_{i}-2 P_{i} B B^{T} X+2 X B B^{T} X-\beta_{i} P_{i} & \left(B^{T} P_{i}-S\right)^{T} \\
B^{T} P_{i}-S & -I
\end{array}\right]<0} \\
& S=M E_{\perp}-B^{T} P_{i} \\
& P_{i}=P_{i}^{T}>0 \\
& {\left[\begin{array}{cc}
A_{j}^{T} P_{i}^{j}+P_{i}^{j} A_{j}-2 X^{j} B_{j} B_{j}^{T} P_{i}^{j}-2 P_{i}^{j} B_{j} B_{j}^{T} X^{j}+2 X^{j} B_{j} B_{j}^{T} X^{j}-\beta_{i} P_{i}^{j} & \left(B_{j}^{T} P_{i}^{j}-S^{j}\right)^{T} \\
B_{j}^{T} P_{i}^{j}-S^{j} & -I
\end{array}\right]<0} \\
& S^{j}=M E_{\perp}-B_{j}^{T} P_{i}^{j} \\
& P_{i}^{j}=P_{i}^{j^{T}}, \quad j=1, \cdots, r
\end{aligned}
$$


TABLE I

Parameter VAlues for Example 2

\begin{tabular}{|c|c|c|c|c|}
\hline Operating point & 1 & 2 & 3 & 4 \\
\hline Mach number & 0.5 & 0.9 & 0.85 & 1.5 \\
\hline Altitude (ft) & 5000 & 35000 & 5000 & 35000 \\
\hline$a_{11}$ & -0.9896 & -0.6607 & -1.702 & -0.5162 \\
\hline$\overline{a_{12}}$ & 17.41 & 18.11 & 50.72 & 26.96 \\
\hline$a_{13}$ & 96.15 & 84.34 & 263.5 & 178.9 \\
\hline$a_{21}$ & 0.2648 & 0.08201 & 0.2201 & -0.6896 \\
\hline$a_{22}$ & -0.8512 & -0.6587 & -1.418 & -1.225 \\
\hline$a_{23}$ & -11.39 & -10.81 & -31.99 & -30.38 \\
\hline$b_{1}$ & -97.78 & -272.2 & -85.09 & -175.6 \\
\hline
\end{tabular}

After 29 iterations, we obtained $K=-2.5709, M=\left[\begin{array}{ll}2.5709 & 0\end{array}\right]$, and

$$
\begin{aligned}
& P_{0}=\left[\begin{array}{rr}
7.5030 & -0.0030 \\
-0.0030 & 0.1521
\end{array}\right] \times 10^{-2} \\
& S_{0}=\left[\begin{array}{ll}
2.4960 & -0.0055
\end{array}\right] \\
& P_{1}=\left[\begin{array}{rr}
1.2929 & -0.0140 \\
-0.0140 & 0.04839
\end{array}\right] \\
& S_{1}=\left[\begin{array}{ll}
1.3162 & -0.1182
\end{array}\right] \\
& P_{2}=\left[\begin{array}{rr}
2.8464 & -0.0004 \\
-0.0004 & 0.0438
\end{array}\right] \times 10^{-2} \\
& S_{2}=\left[\begin{array}{ll}
2.5425 & -0.0019
\end{array}\right] .
\end{aligned}
$$

The eigenvalues are $-40.5506,-1.0203 ;-0.7855 \pm 5.1388 i$; and $-113.0837,-0.4872$, respectively. Hence, the above output feedback gain stabilizes simultaneously the plants.

Example 2: Consider an F4E fighter aircraft model considered in [9]-[12]

$$
\dot{x}(t)=\left[\begin{array}{ccc}
a_{11} & a_{12} & a_{13} \\
a_{21} & a_{22} & a_{23} \\
0 & 0 & -30
\end{array}\right] x+\left[\begin{array}{c}
b_{1} \\
0 \\
30
\end{array}\right] u .
$$

The values of the parameters $a_{11}, a_{12}, a_{13}, a_{21}, a_{22}, a_{23}$, and $b_{1}$ at different operating points are given in Table I.

Now we consider simultaneously stabilizing the four plants at different operating points using the ILMI Algorithm. After one iteration with $R=1$, a solution is found with

$$
\beta=-3.6021, \quad K=\left[\begin{array}{lll}
0.9865 & 7.8075 & -6698
\end{array}\right] * 10^{4}
$$

while after 24 iterations

$$
\begin{aligned}
K & =\left[\begin{array}{lll}
0.0218 & 0.2391 & -0.1180
\end{array}\right] \\
M & =\left[\begin{array}{lll}
-0.0218 & -0.2391 & 0.1180
\end{array}\right] \\
P_{1} & =\left[\begin{array}{lll}
0.1530 & 1.1928 & 0.0577 \\
1.1928 & 9.7085 & 0.1447 \\
0.0577 & 0.1447 & 0.3292
\end{array}\right] \times 10^{-3} \\
P_{2} & =\left[\begin{array}{rrr}
0.0193 & 0.2261 & -0.0443 \\
0.2261 & 2.7315 & -0.5142 \\
-0.0443 & -0.5142 & 0.1679
\end{array}\right] \times 10^{-3} \\
P_{3} & =\left[\begin{array}{rrr}
0.0350 & 0.4508 & -0.1586 \\
0.4508 & 6.4330 & -2.6305 \\
-0.1585 & -2.6305 & 1.3205
\end{array}\right] \times 10^{-3} \\
P_{4} & =\left[\begin{array}{rrr}
0.0371 & 0.1361 & 0.1143 \\
0.1361 & 2.6636 & -1.5783 \\
0.1143 & -1.5783 & 2.2186
\end{array}\right] \times 10^{-3} \\
S_{1} & =\left[\begin{array}{lll}
-0.0086 & -0.1269 & 0.1138
\end{array}\right]
\end{aligned}
$$

$$
\begin{aligned}
& S_{2}=\left[\begin{array}{lll}
-0.0152 & -0.1622 & 0.1009
\end{array}\right] \\
& S_{3}=\left[\begin{array}{lll}
-0.0141 & -0.1219 & 0.0649
\end{array}\right] \\
& S_{4}=\left[\begin{array}{lll}
-0.0187 & -0.1679 & 0.0715
\end{array}\right] \text {. }
\end{aligned}
$$

The eigenvalues of the four closed-loop systems are -0.7236 , $-33.1021,-3.6899 ;-33.5600,-5.3834,-1.8588 ;-32.5675$, $-2.9751 \pm 3.6091 i$; and $-3.5694 \pm 5.3054 i,-31.9763$, respectively. Hence, this state feedback simultaneously stabilizes these four plants.

Now we assume the states are not measurable directly and the output matrix is given by

$$
C=\left[\begin{array}{rrr}
1 & 2 & 0 \\
1 & 0 & -2
\end{array}\right]
$$

In this case, the third plant is nonminimum phase. The zeros are $19.2431 \pm 11.1025 i$ and $12.6260,22.1417$. Using the above algorithm, $\beta=-0.0427$ is obtained after 41 iterations. To achieve a smaller gain, the algorithm is allowed to continue iterating, only after another three iterations, and we get

$$
\begin{aligned}
K & =\left[\begin{array}{lll}
0.1109 & -0.0952
\end{array}\right] \\
M & =\left[\begin{array}{lll}
1.2874 & -0.6437 & 0.6437
\end{array}\right] \\
P_{1} & =\left[\begin{array}{ccc}
0.1879 & 1.1558 & 0.0435 \\
1.1558 & 10.0022 & 0.1725 \\
0.0435 & 0.1725 & 1.0490
\end{array}\right] * 10^{-3} \\
P_{2} & =\left[\begin{array}{ccc}
0.0194 & 0.2780 & 0.0084 \\
0.2780 & 3.9787 & 0.09431 \\
0.0084 & 0.0943 & 0.9503
\end{array}\right] * 10^{-3} \\
P_{3} & =\left[\begin{array}{ccc}
0.0360 & 0.5208 & -0.1426 \\
0.5208 & 7.6748 & -2.7429 \\
-0.1426 & -2.7429 & 5.7825
\end{array}\right] * 10^{-3} \\
P_{4} & =\left[\begin{array}{ccc}
0.0509 & 0.2021 & 0.3420 \\
0.2021 & 4.0981 & -1.0280 \\
0.3420 & -1.0280 & 5.2243
\end{array}\right] * 10^{-3} \\
S_{1} & =\left[\begin{array}{lll}
0.001426 & -0.1139 & -0.2177
\end{array}\right] \\
S_{2} & =\left[\begin{array}{lll}
-0.0106 & -0.1489 & -0.2167
\end{array}\right] \\
S_{3} & =\left[\begin{array}{lll}
-0.008302 & -0.0952 & -0.3761
\end{array}\right] \\
S_{4} & =\left[\begin{array}{lll}
-0.01697 & -0.1554 & -0.2872
\end{array}\right] .
\end{aligned}
$$

The eigenvalues of the four closed-loop systems are -0.7714 , $-22.2118,-4.6724 ;-6.9586,-1.8756,-21.0284 ;-4.4036 \pm$ $2.6944 i,-19.9292$; and $-4.9677 \pm 5.1719 i,-18.8380$, respectively. Hence, this output feedback simultaneously stabilizes these four plants.

\section{CONCLuding Remarks}

The main point of this paper is to propose a numerical algorithm to solve the problem of simultaneous stabilization via static output feedback and state feedback control. A necessary and sufficient condition for simultaneous stabilizability of a collection of MIMO plants via static output feedback is given using a collection of coupled ARE's and ARI's. The corresponding result for the state feedback case is also derived. The simultaneous stabilization problem is recast as a computational procedure. An iterative algorithm based on the LMI technique has been presented. Although the issue of convergence is yet to be established, the effectiveness of the approach is demonstrated well by numerical examples involving this class of BMI problems. 


\section{ACKNOWLEDGMENT}

The authors would like to thank the Associate Editor and anonymous reviewers for their constructive comments and suggestions.

\section{REFERENCES}

[1] T. E. Djaferis, "To stabilize a $k$ real parameter affine family of plants it suffices to simultaneously stabilize $4^{k}$ polynomials," Syst. Contr. Lett., vol. 16, pp. 187-193, 1991.

[2] R. Saeks and J. Murray, "Fractional representation algebraic geometry and the simultaneous stabilization problem," IEEE Trans. Automat. Contr., vol. 27, pp. 895-903, 1982.

[3] M. Vidyasagar and N. Viswanadham, "Algebraic design techniques for reliable stabilization," IEEE Trans. Automat. Contr., vol. 27, pp. 1085-1095, 1982.

[4] M. Vidyasagar, Control System Synthesis: A Factorization Approach. Cambridge, U.K.: MIT Press, 1985.

[5] K. Wei, "The solution of a transcendental problem and its applications in simultaneous stabilization problems," IEEE Trans. Automat. Contr., vol. 37, pp. 1305-1315, 1992.

[6] J. H. Chow, "Pole-placement design approach for systems with multiple operating conditions," IEEE Trans. Automat. Contr., vol. 35, pp. 278-288, 1990

[7] H. Chen, J. H. Chow, M. A. Kale, and K. D. Minto, "Simultaneous stabilization using stable system inversion," Automatica, vol. 31 , no. 4 pp. 531-542, 1995.

[8] V. Blondel and M. Gevers, "Simultaneous stabilizability of three linear systems is rationally undecidable," Math. Contr. Sig. Syst., vol. 6, pp. 135-145, 1993.

[9] D. N. Wu, W. B. Gao, and M. Chen, "Algorithm for simultaneous stabilization of single-input systems via dynamic feedback," Int. J. Contr., vol. 51, pp. 631-642, 1990.

[10] G. D. Howitt and R. Luus, "Simultaneous stabilization of linear singleinput systems by linear state feedback control," Int. J. Contr., vol. 54 , pp. 1058-1072, 1991.

[11] _ "Control of a collection of linear systems by linear state feedback control," Int. J. Contr., vol. 58, pp. 79-96, 1993.

[12] M. Paskota, V. Sreeram, K. L. Teo, and A. I. Mees, "Optimal simultaneous stabilization of linear single-input systems via linear state feedback control," Int. J. Contr., vol. 60, pp. 483-498, 1994.

[13] J. C. Geromel, P. L. D. Peres, and S. R. Souza, "On a convex parameter space method for linear control design of uncertain systems," SIAM J. Contr. Optim., vol. 29, no. 2, pp. 381-402, 1991.

[14] S. Boyd, L. El Ghaoui, E. Feron, and V. Balakrishnan, Linear Matrix Inequalities in System and Control Theory. Philadelphia, PA: SIAM, 1994.

[15] B. D. O. Anderson and J. B. Moore, Optimal Control: Linear Quadratic Methods. Englewood Cliffs, NJ: Prentice Hall, 1990.

[16] J. C. Geromel, P. L. D. Peres, and S. R. Souza, "Convex analysis of output feedback control problems: Robust stability and performance," IEEE Trans. Automat. Contr., vol. 41, pp. 997-1002, 1996.

[17] D. Cheng and C. F. Martin, "Boundaries of conditional quadratic forms-A comment on 'Stabilization via static output feedback,", IEEE Trans. Automat. Contr., vol. 40, pp. 500-502, 1995.

[18] V. Kucera and C. de Souza, "A necessary and sufficient condition for output feedback stabilizability," Automatica, vol. 31, no. 9, pp. $1357-1359,1995$

[19] A. Trofino-Neto and V. Kucera, "Stabilization via static output feedback," IEEE Trans. Automat. Contr., vol. 38, pp. 764-765, 1993.

[20] T. Iwasaki and R. E. Skelton, "Parametrization of all stabilizing controllers via quadratic Lyapunov functions," J. Optim. Theory Appl., vol. 85, pp. 291-307, 1995.

[21] T. Iwasaki, R. E. Skelton, and J. C. Geromel, "Linear quadratic suboptimal control with static output feedback," Syst. Contr. Lett., pp. 421-430, 1994

[22] P. Gahinet, A. Nemirovski, A. J. Laub, and M. Chilali, LMI Control Toolbox. Natick, MA: The MathWorks, 1995.

[23] A. A. Pimpalkhare and B. Bandyopadhyay, "Comments on 'Stabilization via static output feedback," IEEE Trans. Automat. Contr. vol. 39, p. 1148, 1994.
[24] Y. Y. Cao, Y. X. Sun, and W. J. Mao, "A new necessary and sufficient condition for static output feedback stabilizability and comment on 'Stabilization via static output feedback' "IEEE Trans. Automat. Contr., vol. 43, pp. 1110-1111, Aug. 1998.

[25] K. M. Grigoriadis and R. E. Skelton, "Alternating convex projection methods for covariance control design," Automatica, vol. 32, no. 8, pp. 1117-1125, 1996.

[26] O. Toker and H. Ozbay, "On the NP-hardness of solving bilinear matrix inequalities and simultaneous stabilization with static output feedback," in Proc. American Contr. Conf., Seattle, WA, 1995.

[27] V. L. Syrmos, C. Abdallah, P. Dorato, and K. Grigoriadis, "Static output feedback: A survey," Automatica, vol. 33, no. 2, pp. 125-137, 1997.

[28] Y.-Y. Cao, J. Lam, and Y.-X. Sun, "Static output feedback stabilization: An ILMI approach," Automatica, vol. 34, no. 12, pp. 1641-1645, 1998

\section{A Parametric Approach for $l_{1}$ Robust Identification}

Shuning Wang, Jianshe Dai, and Masahiro Tanaka

Abstract-A convergent algorithm for $l_{1}$ robust identification of a stable rational transfer function with known order is proposed, which is implemented by solving a linear programming problem and can produce a rational transfer function with a fixed order. An explicit upper bound on its worst case identification error is given. Its performance is proven to be close to that of an interpolatory algorithm if a high-order Galois sequence is taken as the input signal.

Index Terms - Control-oriented models, linear programming, parameter identification, robust estimation, transfer function.

\section{INTRODUCTION}

The problem considered in this paper may be regarded as a novel formulation within the framework of robust control oriented worst case/deterministic system identification, which was first posed in [1] and then studied by many authors such as [2]-[6]. In this framework, the a priori information for system identification consists of a lower bound on the relatively stability of the plant, an upper bound on a certain gain associated with the plant, and an upper bound on the noise level. The task of identification is to design an algorithm which may map a group of observed data, either in frequency domain or in time domain, onto a nominal transfer function, and to derive an explicit upper bound on its worst case/deterministic identification error measured by $H_{\infty}$ or $l_{1}$ norm. A number of references concerning this subject may be found in the survey paper [7].

It is worth noting that all the works mentioned above tackle this problem with a nonparametric approach, which takes the transfer function to be identified as an infinite Taylor's expansion. Though this approach may avoid making any assumption on the order of a

Manuscript received December 2, 1996; revised July 15, 1997. Recommended by Associate Editor, J. Chen. This work was supported in part by the 863 Program of China under Grant 863-512-9505-17 and the Science Foundation of Tsinghua University.

S. Wang is with the Department of Automation, Tsinghua University, Beijing, 100084 China.

J. Dai is with the Institute of Systems Engineering, Huazhong University of Science and Technology, Wuhan, 430074 China.

M. Tanaka is with the Department of Information Technology, Okayama University, Okayama, 700-8530 Japan (e-mail: m.tanaka@ computer.org).

Publisher Item Identifier S 0018-9286(99)03030-5. 